\title{
Nutrición para la salud y el estado físico: azúcar y otros edulcorantes ${ }^{1}$
}

\author{
Wendy Dahl y Linda Bobroff; traducido por Daniela Rivero Mendoza ${ }^{2}$
}

\section{¿Por qué la preocupación?}

La mayoría de las personas disfruta el dulce sabor del azúcar. De hecho, los bebés muy pequeños sonríen cuando se les pone una gota de agua azucarada en los labios. Sin embargo, los azúcares proporcionan calorías, pero ninguno de los nutrientes que necesitamos para una buena salud. Las personas que comen demasiados alimentos con alto contenido de azúcares a menudo consumen más calorías y menos nutrientes de los que necesitan. Esto puede conducir a la obesidad y a una variedad de problemas de salud. Existe una fuerte evidencia de que la mayoría de los estadounidenses serían más saludables si consumieran menos alimentos y bebidas que contienen azúcares añadidos. Veamos por qué la ingesta excesiva de azúcares agregados está causando problemas de salud para muchos estadounidenses.

- El azúcar es una fuente de calorías "vacías". Comer o beber un exceso de calorías puede contribuir a la obesidad y los problemas de salud asociados.

- Los alimentos y bebidas con alto contenido de azúcares agregados a menudo reemplazan los alimentos y bebidas más saludables en la dieta. Esto puede resultar en una dieta pobre en nutrientes que no contribuye a la buena salud.
- El azúcar contribuye a la caries dentales, que es un importante problema de salud pública en los Estados Unidos, especialmente entre las familias de bajos ingresos.

Esta publicación proporciona información sobre azúcares y otros edulcorantes en la dieta estadounidense. La publicación describe fuentes ocultas de azúcares agregados en los alimentos para ayudar a aquellos que desean reducir la ingesta de azúcar, a encontrar los azúcares agregados en sus dietas. La sección sobre edulcorantes de alta intensidad analiza las características de cada edulcorante aprobado.

\section{¿Qué es el azúcar?}

El azúcar es un tipo de carbohidrato. Los carbohidratos son uno de los nutrientes que proporcionan calorías en nuestras dietas. Los otros nutrientes que contienen calorías son las proteínas y las grasas. Existen varios tipos de azúcares, todos los cuales proporcionan aproximadamente cuatro calorías por gramo, lo mismo que otros carbohidratos y proteínas. Todos los azúcares son edulcorantes nutritivos, lo que significa que son dulces y aportan calorías. Los edulcorantes que proporcionan pocas calorías o ninguna y que utilizamos como sustitutos del azúcar se conocen como edulcorantes (endulzantes) de alta intensidad.

1. Este documento, FSHN20-46s, es uno de una serie de publicaciones del Food Science and Human Nutrition, Servicio de Extensión Cooperativa de la Florida, Instituto de Alimentos y Ciencias Agrícolas, Universidad de la Florida. (UF/IFAS). Fecha de primera publicación: octubre 2020. Visite nuestro sitio web EDIS en https://edis.ifas.ufl.edu.

2. Wendy Dahl, associate professor, Food Science and Human Nutrition Department; $y$ Linda Bobroff, professor emeritus, Department of Family, Youth and Community Sciences; traducido por Daniela Rivero Mendoza, Extension and research coordinator; UF/IFAS Extension, Gainesville, FL 32611.

The Institute of Food and Agricultural Sciences (IFAS) is an Equal Opportunity Institution authorized to provide research, educational information and other services

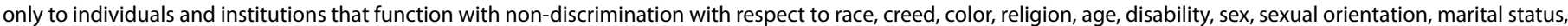

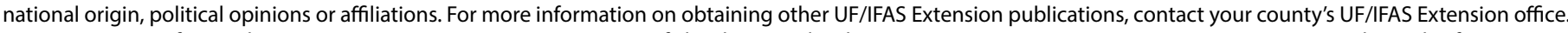
U.S. Department of Agriculture, UF/IFAS Extension Service, University of Florida, IFAS, Florida A \& M University Cooperative Extension Program, and Boards of County Commissioners Cooperating. Nick T. Place, dean for UF/IFAS Extension. 


\section{Edulcorantes Nutritivos}

El edulcorante nutritivo más común es el azúcar de mesa o la sacarosa. La sacarosa es un azúcar doble (disacárido) compuesto de fructosa y glucosa, dos azúcares simples (monosacáridos). La fructosa y la glucosa se encuentran naturalmente en las frutas y la miel. La sacarosa también se refina a partir de la remolacha azucarera o la caña de azúcar y se encuentra en una amplia variedad de alimentos procesados, algunos de los cuales (por ejemplo, aderezos para ensaladas y kétchup, también conocida como salsa de tomate), ¡ni siquiera son dulces!

La fructosa es más dulce que otros azúcares y se usa para endulzar muchos alimentos. Debido a que es tan dulce, se puede usar una cantidad menor, lo que agrega menos calorías. Sin embargo, la fructosa no tiene un sabor más dulce que otros azúcares en todos los alimentos y bebidas, y es más costosa que la sacarosa.

La lactosa, o azúcar de la leche, es otro disacárido, que contiene los monosacáridos glucosa y galactosa. La lactosa proporciona un pequeño porcentaje de la ingesta de azúcar en la dieta estadounidense. Como puede ver por el sabor de la leche, la lactosa no es un azúcar muy dulce.

Otro azúcar que se encuentra en una variedad de alimentos es el disacárido maltosa, que consta de dos moléculas de glucosa. La maltosa se usa para elaborar cerveza y está formada por levadura en la fabricación de pan. Las legumbres (frijoles y lentejas) y los cereales contienen pequeñas cantidades de maltosa.

Tabla 1. Tipos comunes de azúcar.

\begin{tabular}{|l|l|}
\hline \multicolumn{1}{|c|}{ Monosacáridos } & \multicolumn{1}{c|}{ Disacáridos } \\
\hline Glucosa (Dextrosa) & Maltosa $=$ Glucosa + Glucosa \\
\hline Fructosa & Sacarosa $=$ Glucosa + Fructosa \\
\hline Galactosa & Lactosa $=$ Glucosa + Galactosa \\
\hline
\end{tabular}

Tabla 2. Dulzura relativa de azúcares y otros edulcorantes (FDA 2015; Mahan y Escott-Stump 2008).

\begin{tabular}{|l|l|}
\hline Advantame (el más dulce) & Fructosa \\
\hline Neotame & Extracto de Luo Han Guo \\
\hline Sucralosa & Sacarosa y xilitol \\
\hline Sacarina & Glucosa \\
\hline Stevia & Sorbitol y manitol \\
\hline Aspartamo y acesulfamo-K & Galactosa y maltosa \\
\hline Ciclamato (prohibido en los EE. UU.) & Lactosa (menos dulce) \\
\hline
\end{tabular}

Las principales fuentes de azúcares agregados en la dieta de los EE. UU. son las bebidas azucaradas (refrescos, bebidas energéticas, bebidas deportivas, café y té azucarado, bebidas alcohólicas y aguas aromatizadas), que proporcionan casi la mitad del total de azúcares agregados y meriendas y dulces (postres a base de granos, postres lácteos como helados, dulces, mermeladas, jarabes, etc.), que proporcionan cerca de un tercio de los azúcares añadidos. Las actuales guías dietéticas para los estadounidenses recomiendan un cambio en los patrones de alimentación para reducir la ingesta de azúcar agregada a menos del $10 \%$ de las calorías (USDHHS y USDA 2015).

El azúcar, los jarabes y otros edulcorantes se encuentran en una variedad de alimentos como ingredientes agregados. Los alimentos con azúcares agregados incluyen dulces, pasteles, tartas, galletas, cereales, bebidas, aderezos para ensaladas y muchos otros. Los profesionales de la salud están preocupados por los azúcares agregados en este tipo de alimentos y otras fuentes de azúcares "ocultas". Las frutas, que contienen azúcares naturales, son parte de una dieta saludable. Proporcionan una variedad de nutrientes y fibra y son excelentes opciones para postres o meriendas (tentempiés o refrigerios) dulces y saludables. La leche también contiene un azúcar natural (lactosa) que es una preocupación solo para aquellos que no pueden digerir este azúcar. La leche sin lactosa es una opción para quienes son intolerantes a la lactosa. El yogur y el queso a menudo son bien tolerados.

Los azúcares agregados juegan un papel versátil en los alimentos. Estos les dan dulzura a los alimentos, una cualidad que muchas personas disfrutan. Además de mejorar el sabor de los alimentos, los azúcares agregan textura y color a los productos horneados y ayudan a espesar, reafirmar o preservar alimentos como pudines, mermeladas y jaleas. Comer estos alimentos con moderación está bien, pero muchas personas consumen mucho más azúcar agregada de lo que es saludable.

\section{¿Cuánto azúcar comemos?}

En los EE. UU., entre el 2003 y el 2010, los azúcares agregados constituyeron aproximadamente el 14\% de la ingesta de calorías de los mayores de seis años. Esto no cuenta las formas naturales de azúcar en frutas y productos lácteos. Los adultos (pero no los niños) con ingresos más bajos tienden a tener una mayor ingesta de azúcares añadidos, lo que puede desempeñar un papel en sus tasas más altas de obesidad (CDC 2019). 


\section{Azúcares y Nutrición}

Quizás se pregunte si el azúcar cruda, la miel o el néctar de agave son más nutritivos que otros azúcares. La respuesta es no. Todos los siguientes edulcorantes son nutricionalmente iguales; contienen calorías junto con ningún (o mínimo) nutriente aparte del azúcar (Duyff 2012).

Néctar de agave: un edulcorante nutritivo producido a partir del corazón de la planta de agave. El jugo fresco se calienta, convirtiendo los carbohidratos complejos en azúcares simples, principalmente fructosa y glucosa.

Azúcar en bruto: cristales gruesos y granulados formados por la evaporación del jugo de caña de azúcar. El azúcar crudo contiene impurezas y no se vende en tiendas. $\mathrm{Cu}-$ ando se eliminan las impurezas junto con la mayoría de la melaza, el azúcar se puede vender como azúcar turbinado.

Melaza: jarabe de color oscuro que es un subproducto de la producción de sacarosa. Contiene hasta $70 \%$ de sacarosa. La melaza proporciona cantidades muy pequeñas de calcio y hierro.

Azúcar moreno: cristales de sacarosa cubiertos con pequeñas pero variadas cantidades de melaza.

Azúcar invertido: mezcla de glucosa y fructosa formada por división química de sacarosa. El azúcar invertido evita la cristalización del azúcar de caña en los dulces.

Azúcar en polvo o repostería: cristales de sacarosa finamente molidos mezclados con una pequeña cantidad de maicena.

Miel: un edulcorante que contiene fructosa, glucosa, maltosa y sacarosa. Está hecho por abejas y contiene solo pequeñas cantidades de vitaminas y minerales.

Jarabe de maíz: un edulcorante hecho de almidón de maíz y utilizado en muchos alimentos preparados comercialmente.

Jarabe de maíz alto en fructosa (JMAF): un edulcorante hecho de almidón de maíz. Un proceso enzimático aumenta ligeramente el contenido de fructosa, lo que hace que el JMAF sea más dulce que el jarabe de maíz normal. El contenido de glucosa y fructosa del JMAF es similar al de la sacarosa (azúcar de mesa).

\section{El azúcar y tu salud}

Muchas personas creen que el azúcar causa una variedad de enfermedades. Sin embargo, no hay buena evidencia de que el consumo de azúcar sea, por sí mismo, un factor significativo en el desarrollo de enfermedades del corazón, diabetes, hipoglucemia (bajo nivel de azúcar en la sangre) o hiperactividad. Sin embargo, dado que comer una gran cantidad de azúcar puede agregar muchas calorías a la dieta, puede contribuir al desarrollo de la obesidad, que es un factor de riesgo para varios otros problemas de salud.

Dependiendo de la cantidad de azúcar consumida, el impacto sobre el total de calorías consumidas variará. Comer una libra de azúcar por semana agregaría más de 1800 calorías por semana, lo que tendría un efecto significativo en el equilibrio calórico. Para muchas personas, reducir el consumo de azúcar es útil para disminuir el consumo de calorías. Junto con la disminución del consumo de alimentos ricos en grasas y el aumento del ejercicio, la reducción de los alimentos con azúcares añadidos puede ser un factor importante para controlar la obesidad.

El azúcar ha reemplazado a algunos carbohidratos complejos en las dietas de muchos estadounidenses. Dado que los carbohidratos complejos están asociados con otros nutrientes necesarios para una buena salud, se nos recomienda incluir alimentos como frijoles y guisantes y panes, pastas y cereales integrales en nuestras dietas. Para aumentar nuestra ingesta de alimentos nutritivos ricos en carbohidratos sin un aumento en las calorías totales, debemos disminuir la cantidad de azúcar que consumimos.

\section{Azúcar y salud dental}

El azúcar es un factor directo en el desarrollo de deterioro dental. El tipo de azúcar consumido y la frecuencia de consumo determinan la probabilidad de que se produzca caries dental. Los alimentos pegajosos con alto contenido de azúcar tienden a permanecer en contacto con los dientes por más tiempo, lo que aumenta la posibilidad de caries. Además, cuanto más a menudo durante el día los dientes están expuestos al azúcar, es más probable que se produzca caries dental. La herencia también juega un papel, ya que algunas personas son más susceptibles a las caries que otras. Para evitar la caries dental, evite comer meriendas azucaradas, y cepíllese los dientes y use hilo dental lo antes posible después de comer.

\section{Azúcar en dietas modificadas}

Muchas personas están preocupadas por su consumo de azúcar debido a afecciones médicas, como la diabetes, que 
podrían requerir que modifiquen su uso de carbohidratos y azúcares refinados. Los niveles de glucosa en sangre se controlan en parte por la acción de la insulina, una hormona producida por el páncreas. Cuando el nivel de glucosa en sangre aumenta, como lo hace después de comer, se estimula el páncreas para que produzca insulina, lo que facilita la absorción de glucosa en las células y el retorno a un nivel de glucosa en sangre apropiado. La diabetes es una enfermedad en la que hay un suministro inadecuado de insulina o la hormona no funciona correctamente. En cualquier caso, las personas con diabetes no pueden regular sus niveles de glucosa en la sangre, $y$ deben controlar su diabetes a través de elecciones de estilo de vida y el uso de medicamentos. Equilibrar la ingesta de carbohidratos durante el día es una parte importante de una dieta saludable para las personas con diabetes.

La hipoglucemia es un término que significa glucosa baja en la sangre. Puede ser un síntoma de diversas enfermedades. Si le preocupa que pueda ser hipoglucémico, consulte a su médico. La mayoría de las personas tienen glucosa en sangre relativamente baja en ciertos momentos durante el día. Por ejemplo, cuando nos levantamos por la mañana, nuestra glucosa en sangre puede ser más baja de lo normal. Sin embargo, un desayuno nutritivo proporciona los carbohidratos necesarios para impulsar el nivel de glucosa en sangre a la normalidad. Cuando su nivel de glucosa en sangre es bajo, puede sentirse cansado, débil y hambriento. Para aliviar estos síntomas, coma alimentos que contengan algunas proteínas y grasas junto con un carbohidrato complejo. El consumo de azúcares simples en alimentos como dulces o refrescos provoca una elevación rápida pero breve de la glucosa en sangre. Esta no es la forma más saludable de satisfacer sus dolores de hambre matutinos.

Tenga en cuenta que cuando la glucosa en sangre de una persona con diabetes disminuye peligrosamente, se trata de una emergencia médica. Esto requiere un aumento rápido de la glucosa en sangre que solo pueden proporcionar los azúcares simples. Las personas con diabetes, especialmente aquellas que toman insulina o un medicamento oral que puede causar hipoglucemia, siempre deben llevar consigo una forma de glucosa para tratar la glucosa baja en la sangre.

\section{Edulcorantes de alta intensidad}

Los edulcorantes de alta intensidad proporcionan alternativas a los azúcares en una variedad de alimentos y bebidas. Se clasifican como edulcorantes nutritivos o edulcorantes no nutritivos (NNS por sus siglas en inglés), dependiendo de si proporcionan o no calorías.

El único edulcorante nutritivo de alta intensidad que está actualmente en el mercado en los Estados Unidos es el aspartamo, comúnmente conocido por su nombre comercial, Nutrasweet ${ }^{\natural}$. Los NNS disponibles en este país son advantame, neotame, sucralosa, glucósidos de stevia (de la planta de stevia), Luo Han Guo o extracto de fruta de monje, sacarina y acesulfamo de potasio (acesulfamo K). Otros NNS que actualmente no están aprobados para su uso en los EE. UU. incluyen el ciclamato, el alimate, la hoja entera y el extracto de stevia crudo, la neohesperidina y la taumatina. Uno o más de estos pueden estar disponibles algún día para su uso en los EE. UU. (CDC 2016; Duyff 2012).

El aspartamo es un edulcorante reducido en calorías ampliamente utilizado y aprobado para su uso en los EE. UU. Aunque el aspartamo proporciona la misma cantidad de calorías por gramo que el azúcar, es aproximadamente 200 veces más dulce que la sacarosa, por lo que se necesita una cantidad muy pequeña para endulzar los alimentos. $\mathrm{Su}$ sabor es similar al de la sacarosa, y no tiene un regusto. El aspartamo se descompone rápidamente a altas temperaturas y es menos estable en líquidos que en alimentos sólidos. Por lo tanto, pueden surgir problemas de sabor cuando las bebidas se almacenan durante largos períodos a altas temperaturas (por ejemplo, mantener una caja de refrescos dietéticos en su garaje caliente).

La Administración de Alimentos y Medicamentos (FDA por sus siglas en inglés) aprobó el aspartamo en 1974, y luego retiró su aprobación cuando surgieron preguntas sobre la seguridad del aspartamo. Después de una audiencia en la corte y una revisión de los estudios toxicológicos que se habían realizado, el aspartamo fue nuevamente aprobado como seguro para su uso en octubre de 1981. Cuando la FDA aprobó el uso del aspartamo en bebidas carbonatadas en 1983, algunos científicos y grupos de consumidores expresaron su preocupación sobre la seguridad de los metabolitos del aspartamo, que incluyen fenilalanina, ácido aspártico, metanol (alcohol de madera) y dicetopiperazina (DKP). A excepción de DKP, estas sustancias se encuentran naturalmente en una variedad de alimentos, o se forman en los alimentos durante la cocción o el procesamiento. DKP se probó a fondo y no se identificaron efectos adversos. En febrero de 1984, la FDA solicitó a los Centros para el Control y la Prevención de Enfermedades (CDC) en Atlanta que investigaran las quejas de los consumidores relacionadas con el consumo de aspartamo. Los CDC concluyeron que algunas personas pueden ser inusualmente 
sensibles a los productos que contienen aspartamo, pero que no hubo evidencia de consecuencias adversas graves para la salud relacionadas con el uso del edulcorante. Las personas con una enfermedad hereditaria, la fenilcetonuria (PKU), no pueden metabolizar la fenilalanina, y sus dietas deben controlarse para evitar el daño cerebral. Por lo tanto, todos los productos que contienen aspartamo deben etiquetarse con una advertencia a las personas con PKU de que el producto contiene fenilalanina.

La sacarina es un NNS que es aproximadamente 200 veces más dulce que la sacarosa. Hasta 1981, era el único NNS disponible en los EE. UU. Después de la prohibición de ciclamatos en 1970. Aunque la sacarina ha estado en el mercado desde principios de 1900, fue investigada por la FDA debido a estudios que indicaron un vínculo entre el consumo de sacarina y Cáncer de vejiga en ratas. Como resultado del estudio original informado a principios de la década de 1970, la FDA eliminó la sacarina de su lista de aditivos alimentarios "Generalmente reconocidos como seguros” (GRAS) (FDA 2017), y en 1977, la FDA propuso la prohibición del uso de sacarina. La protesta pública que siguió a esta propuesta llevó a la FDA a suspender la prohibición hasta que se pueda realizar una investigación adicional. El Congreso prorrogó la moratoria cada dos años hasta 1991. Durante años, los alimentos y bebidas que contenían sacarina debían tener una etiqueta de advertencia en el paquete. La Ley de derogación del aviso de sacarina eliminó este requisito en 1996. La sacarina es más conocida como el edulcorante Sweet'N Low ${ }^{\oplus}$, pero más recientemente también se ha vendido bajo los nombres Sweet Twin ${ }^{\oplus} \mathrm{y}$ Necta Sweet ${ }^{\oplus}$.

El acesulfamo K (acesulfamo de potasio) fue aprobado para su uso en categorías específicas de alimentos y bebidas por la FDA en julio de 1988, y como edulcorante de uso general y potenciador del sabor en los alimentos en 2003. Se comercializa como Sunette y Sweet One $^{\circledast}$. El acesulfamo $\mathrm{K}$ no cambia en el cuerpo y no proporciona calorías ni potasio. Como no se descompone a altas temperaturas, este edulcorante se puede usar para cocinar y hornear. Es aproximadamente 200 veces más dulce que el azúcar.

La sucralosa es un NNS de alta intensidad popular debido a la forma en que se vierte y mide como el azúcar para hornear. La sucralosa no se metaboliza en el cuerpo, por lo que no aporta calorías cuando se consume. En 1998, la sucralosa fue aprobada como edulcorante de mesa; en 1999, se aprobó más como edulcorante de uso general. La sucralosa se vende bajo la marca Splenda ${ }^{\oplus}$. Antes de la aprobación, la FDA revisó más de 110 estudios para garantizar la seguridad en el consumo. La sucralosa se encuentra en bebidas, postres congelados, gelatinas, chicles, productos horneados y muchos más alimentos.

Advantame es el NNS de alta intensidad aprobado más recientemente, solo recibió la aprobación de la FDA en 2014, como edulcorante y potenciador general del sabor en los alimentos. Aunque el Advantame en sí mismo es un derivado del aspartamo, tan poco de este edulcorante se usa a la vez que la FDA no requiere una etiqueta de advertencia sobre la presencia de fenilalanina en personas con PKU. Advantame es 20,000 veces más dulce que la sacarosa y es estable al calor. Hasta abril del 2020, Advantame no estaba disponible para la venta comercial bajo una marca o en ningún producto alimenticio en los EE. UU.

Neotame se vende bajo la marca Newtame ${ }^{\circledR}$. De 7,000 a 13,000 veces más dulce que el azúcar, el neotame recibió la aprobación de la FDA en 2002 como edulcorante de alta intensidad y potenciador del sabor en los alimentos. Es parcialmente absorbido por el intestino delgado y se metaboliza rápidamente antes de ser completamente excretado del cuerpo. Al igual que el Advantame, el neotame contiene fenilalanina en una concentración tan pequeña que la cantidad real liberada en el cuerpo es insignificante. Neotame no cambia la estructura a altas temperaturas, lo que hace que este producto sea apropiado para hornear.

Los glucósidos de Stevia son NNS de alta intensidad hechos de la planta Stevia rebaudiana Bertoni, más comúnmente conocida como Stevia. En 1998, la FDA aprobó los glucósidos de stevia para su uso como aditivo alimentario que generalmente se reconoce como seguro (GRAS). Los productos en la lista GRAS son aditivos alimentarios que cumplen con un estándar de seguridad establecido por la FDA con una "certeza razonable de que no hay daños" en uso. Las sustancias GRAS se someten a una revisión previa a la comercialización, al igual que otros aditivos alimentarios, pero la evaluación la realizan expertos que examinan la seguridad del producto con datos científicos disponibles al público. Para una sustancia alimenticia (como la sacarina) que fue certificada GRAS antes de 1958, su uso se considera seguro "a través de la experiencia basada en el uso común en los alimentos" (FDA 2019).

Actualmente, el rebaudiósido A purificado y el esteviósido están aprobados bajo la certificación GRAS. Las hojas enteras de stevia no están aprobadas como NNS, pero pueden venderse como un suplemento dietético. La stevia es estable en almacenamiento en forma seca y más estable que el acesulfamo $\mathrm{K}$ y el aspartamo en forma líquida. 
El extracto de Luo Han Guo es otro producto GRAS aprobado para su uso por la FDA en 2009. Es el nombre común del compuesto Siraitia grosvenorii o extracto de fruta Swingle. El nivel de dulzura varía de 150 a 300 veces más dulce que el azúcar, dependiendo de los glucósidos utilizados. Un nombre comúnmente reconocido para este producto es la fruta del monje, que se ha utilizado durante siglos en China. Se sabe que el extracto de Luo Han Guo tiene un regusto a altos niveles.

El ciclamato es un NNS que es aproximadamente 30 veces más dulce que la sacarosa. Era un edulcorante popular en las bebidas carbonatadas en los EE. UU. En la década de 1960, a menudo utilizado en combinación con sacarina. El ciclamato fue prohibido en los EE. UU. En 1970 porque los estudios en ratas implicaron que era un carcinógeno (un agente cancerígeno). La validez de los datos ha sido cuestionada desde entonces, pero el ciclamato no está disponible en este país. Sin embargo, actualmente se comercializa en unos 40 países, incluido Canadá. En 1984, el Comité de Evaluación del Cáncer de la FDA informó que la evidencia disponible indicaba que el ciclamato no es cancerígeno. El Consejo Nacional de Investigación de la Academia Nacional de Ciencias declaró en 1985 que el ciclamato no es cancerígeno e indicó que no se necesita más investigación.

\section{Ingesta de edulcorantes no nutritivos y salud}

En una revisión de estudios (Toews et al. 2019) se evaluaron los estudios que comparaban los resultados de salud de adultos o niños sanos con una ingesta más baja de NNS en comparación con una mayor ingesta de NNS. Un limitado número de pequeños estudios de intervención en adultos mostró una pequeña reducción del índice de masa corporal y glucosa en sangre en ayunas en individuos que consumen NNS, pero no en aquellos que consumen sacarosa. Aunque varios de los estudios de menor duración no encontraron una reducción significativa en el consumo de energía promedio en adultos que reciben NNS en comparación con los que reciben azúcar (Reid, Hammersley y Duffy 2010, Reid et al. 2013, Reid et al. 2007), un estudio más largo de 10 semanas mostró una menor ingesta de energía en quienes consumen NNS (Raben et al. 2001), lo que puede deberse a que la ingesta diaria de azúcar es menor en quienes consumen NNS (Raben et al. 2001, Reid, Hammersley y Duffy 2010, Reid et al. 2013). En los niños, se observó un aumento menor en el índice de masa corporal con la ingesta de NNS en comparación con la ingesta de azúcar (Toews et al. 2019). Se concluyó que la ingesta de NNS (en comparación con cualquier tipo de azúcar, no intervención, placebo o agua corriente), parecía no mejorar la mayoría de los resultados de salud (es decir, niveles de insulina en sangre, resistencia a la insulina, función pancreática, salud oral, cáncer, enfermedad cardiovascular, enfermedad renal crónica, asma, alergias, estado de ánimo y neurocognición). Sin embargo, de los pocos estudios identificados para cada resultado de salud, la mayoría fueron de corta duración, tuvieron pocos participantes en el estudio y otras limitaciones. Se necesita más investigación para determinar si existe una relación entre la ingesta de NNS y la salud.

De reciente interés son las posibles relaciones entre la ingesta de NNS, la microbiota intestinal y la salud. Dada su dulzura de alta intensidad, la mayoría de los NNS se consumen en cantidades muy bajas y se absorben en el intestino delgado (Ruiz-Ojeda et al. 2019), por lo que no se espera que ejerzan ningún efecto directo sobre la microbiota intestinal que se encuentra en mayor abundancia en el colon. Como se esperaba, hay muy poca evidencia que sugiera que NNS module la microbiota intestinal humana (Ruiz-Ojeda et al. 2019).

\section{Lo que revelan las etiquetas de los alimentos}

La etiqueta de información nutricional actualmente en uso muestra la cantidad total de azúcar en un producto, pero no distingue entre azúcares naturales y agregados. La nueva etiqueta de alimentos aprobada por la FDA en 2016 proporciona esta información importante. Algunos paquetes de alimentos ya cuentan con el nuevo panel de información nutricional, mientras que otros tienen hasta Julio del 2021 para cumplir. Para aquellos paquetes de alimentos que usan el panel de información nutricional anterior, tenga el hábito de leer la lista de ingredientes, que enumera azúcares específicos como ingredientes. Si uno de los términos que significa azúcar se encuentra primero o segundo en la lista de ingredientes, entonces sabe que el azúcar es uno de los principales ingredientes en peso (mire el ejemplo de la lista de ingredientes abajo). El sufijo -ose indica que la sustancia es un azúcar. Tenga en cuenta que no todos los azúcares tienen un nombre que termina en -ose.

Los azúcares son ingredientes en todos los alimentos dulces, pero también se encuentran en otros alimentos, incluidas las mezclas de recubrimiento de carne y pollo, cremas de café no lácteas y aderezos para ensaladas, entre muchos otros alimentos e ingredientes preparados. Los edulcorantes nutritivos en los alimentos pueden aparecer en la etiqueta como edulcorantes de maíz o edulcorantes 
naturales. Un edulcorante natural podría ser la miel, la melaza o el azúcar de dátiles.

\section{Ejemplo: Lista de ingredientes para bebida de desayuno}

INGREDIENTES: leche descremada en polvo, azúcar*, cacao, suero lácteo dulce, sólidos de jarabe de maíz ${ }^{*}$, caseinato de calcio, lactosa ${ }^{\star}$, proteína de soja aislada, caseinato de sodio, lecitina, hidróxido de magnesio, carragenina, sabor a vainilla artificial, ascorbato de sodio, ortofosfato férrico, acetato de vitamina $\mathrm{E}$, palmitato de vitamina A, niacinamida, gluconato de cobre, óxido de zinc, pantotenato de calcio, mononitrato de tiamina, clorhidrato de piridoxina, ácido fólico.

${ }^{\star}$ Fuente de azúcar.

\section{Romper el hábito del azúcar}

Una vez que una persona desarrolla un hábito alimenticio o un deseo de comer, puede ser difícil cambiar ese deseo. Una forma de reducir la ingesta de azúcar es desarrollar gradualmente un gusto por los alimentos que están libres de azúcares bajos o bajos. Por ejemplo, aprenda a disfrutar el sabor de las frutas frescas sin azúcar. Disminuya gradualmente la cantidad de azúcar que agrega al té y al café, y seleccione alimentos y bebidas envasados, como cereales para el desayuno, aderezos para ensaladas y jugos de frutas, que contengan menos azúcar agregada.

Lo mismo ocurre con el azúcar agregado al preparar y cocinar alimentos. Si deja de agregar azúcar a la ensalada de col, puré de manzana y vegetales cocidos, es posible que le gusten sus sabores naturales. Con el tiempo, usted y su familia pueden descubrir que puede eliminar la mayor parte del azúcar agregada de su dieta.

Aquí hay algunas sugerencias para que sea más consciente de sus hábitos de consumo de azúcar y para ayudarlo a romperlos:

- Conozca los alimentos dulces que come. Durante los próximos días, escriba lo que come y ponga una marca de verificación junto a los alimentos dulces. Observe también cuándo y por qué come estos alimentos dulces. ¿Comes dulces por aburrimiento? Trata de disminuir el consumo de alimentos dulces y selecciona opciones más ricas en nutrientes.

- Haga una lista de alimentos que puede sustituir por dulces. Por ejemplo, en lugar de refrescos, puede beber leche, jugo de fruta 100\% natural, agua o té sin azúcar (helado, con limón); en lugar de pastel, galletas o panecillos dulces, pruebe con pan integral, fruta o rebanadas de queso bajas en grasa; y en lugar de dulces, merienda en trozos de frutas o vegetales.

- Busque sus archivos de recetas y libros de cocina existentes. Muchas recetas usan muy poca azúcar y otras pueden modificarse fácilmente. Puede disminuir la cantidad de azúcar en un tercio sin afectar significativamente el sabor o la textura del producto en muchas recetas.

- Lea las etiquetas para estimar el contenido de azúcar. Además del azúcar, busque las palabras dextrosa, maltosa, sacarosa, fructosa, miel, edulcorante de maíz y jarabe de maíz, todas ellas significan azúcar. Si estos aparecen en los primeros tres ingredientes, el producto tiene un alto contenido de azúcar. Además, si varios de estos ingredientes diferentes aparecen en otra parte de la lista, tenga en cuenta que, si los agrega todos, el azúcar podría ser el ingrediente principal (consulte la Tabla 3).

- Compre menos alimentos con alto contenido de azúcar. Si no están en los armarios o en el refrigerador, no se comerán con tanta frecuencia.

- Sustituya los alimentos saludables por algunos de los alimentos con alto contenido de azúcar que normalmente consume. Asegúrese de incluir diariamente una variedad de alimentos de los cinco grupos de alimentos.

- Evite sustitutos pobres. No reemplace el azúcar con alimentos ricos en grasas, bebidas alcohólicas.

\section{Buen sabor con menos azúcar}

Hay muchas maneras de reducir la cantidad de azúcar que se consume sin renunciar al sabor que le gusta. Sea creativo y experimente. Aquí hay 28 ideas sabrosas para comenzar:

\section{Bebidas}

- Sustituya el jugo $100 \%$ de fruta mezclado con soda club por refrescos, cócteles de jugo de fruta, ponches y otras bebidas que contengan grandes cantidades de azúcar.

- Pruebe el té sin azúcar con una rodaja de limón o una ramita de menta fresca.

- Disfrute de agua, agua mineral o gaseosa con una rodaja de lima o limón fresco.

- Reduzca el consumo de refrescos que contienen azúcar; en su lugar, elija jugos de fruta $100 \%$ (en pequeñas cantidades), té helado sin azúcar, suero de leche y leche baja en grasa.

- Agregue una ramita de canela o una rodaja de naranja al café sin azúcar. 


\section{Leche y Productos Lácteos}

- Sustituya el yogur natural por yogur con sabor a fruta y agregue su propia fruta fresca y una pizca de canela o nuez moscada para darle sabor.

- Elija leche sin sabor sobre otros sabores como leche con chocolate, que generalmente tienen más azúcar.

\section{Panes, cereales y productos horneados}

- Coma menos galletas, tortas y pasteles. Cuando los tenga, prefiera galletas Graham en lugar de otro tipo de galletas, pasteles de frutas en lugar de otro tipo de pasteles, y pasteles regulares con cobertura de frutas en lugar de tortas glaseadas. Coma porciones pequeñas.

- Agregue fruta fresca o pasas en lugar de azúcar a los cereales para el desayuno simples y listos para comer.

- Haga pasteles sin glaseado o glasee solo la parte superior.

- Use panes de levadura en lugar de pan dulce para las fiestas. Hay una variedad para elegir y usan menos azúcar que los panes tradicionales de vacaciones.

- Experimente con recetas. Disminuya gradualmente entre un cuarto y un tercio la cantidad de azúcar requerida en los productos horneados hasta que obtenga un producto aceptable. Destaque el sabor de los edulcorantes con extracto de vainilla, limón o almendras.

- Use coberturas de fruta fresca o puré de manzana sin azúcar para panqueques, waffles y tostadas francesas en lugar de jarabe o miel.

\section{Alimentos proteicos y platos principales}

- Deje a un lado el azúcar en los platos asiáticos y otros salteados. Es dudoso que incluso note la diferencia.

- Haga sus propias mezclas de empanizado y recubrimiento para carne y pollo. Algunos preparados comercialmente contienen más del 50\% de azúcar.

- Muela su propia mantequilla de maní (muchas tiendas de comestibles tienen máquinas para esto) o busque mantequilla de maní sin azúcar agregada.

\section{Frutas}

- Seleccione frutas frescas, frutas enlatadas en su propio jugo o frutas enlatadas en almíbar "lite" o "extra-lite" en lugar de jarabe pesado.

- Use frutas en lugar de azúcar para endulzar los alimentos. La piña, las pasas, los plátanos, las naranjas y los jugos de frutas sin azúcar se pueden usar en algunos vegetales y platos principales, así como en postres.

- Disfrute de fruta fresca de temporada como un alimento básico en su dieta.

\section{Vegetales}

- No agregue azúcar a las verduras cuando cocine. Para un sabor extra, pruebe hierbas y especias, o en algunos casos, frutas y jugos $100 \%$ de frutas.

- Lea las etiquetas de las verduras congeladas empacadas en salsas o paquetes especiales. Muchos de estos contienen azúcar agregada. Lea las etiquetas de las verduras enlatadas también.

\section{Meriendas (refrigerios o tentempiés)}

- Sustituya las palomitas de maíz, las verduras crudas y las frutas frescas por dulces y otros bocadillos dulces. Haga que estos alimentos saludables sean fáciles de encontrar y comer. Prepárelos con anticipación y guárdelos en el refrigerador en un recipiente de plástico hermético etiquetado como "bocadillos".

- Haga sus propias salsas, ya que las preparadas comercialmente a menudo contienen azúcar. Use vegetales crudos como platos en lugar de galletas saladas, que a menudo también contienen azúcar.

\section{Condimentos}

- Intente hacer su propio aderezo para ensaladas. Muchos apósitos comerciales, tanto mezclas embotelladas como empaquetadas, contienen una gran cantidad de azúcar.

- Pruebe el cardamomo, el cilantro, la albahaca, la nuez moscada, la canela y el jengibre para obtener un sabor ligero y dulce.

- Lea las etiquetas de las salsas embotelladas y las mezclas de condimentos envasadas. Puede hacer fácilmente una versión similar en casa sin el azúcar agregado.

\section{Postres}

- Acostúmbrese a servir frutas frescas o simplemente preparadas para el postre. Una compota de frutas en jugo de naranja, ambrosía, toronja asada o plátano con canela son deliciosas delicias.

- Limite la frecuencia de los alimentos dulces que se comen. Comer dos galletas en la cena será menos dañino para los dientes que una comida por la tarde y la otra por la noche.

Para obtener más información, comuníquese con su agente local de UF / IFAS Extension Family and Consumer Sciences. La oficina de Extensión de UF / IFAS de su condado puede aparecer en las páginas gubernamentales de su directorio telefónico. En Florida, puede encontrar su oficina local de Extensión de UF / IFAS en línea en http://sfyl.ifas. ufl.edu/find-your-local-office/. 


\section{Referencias}

Centers for Disease Control and Prevention (CDC). 2019. "Know Your Limit for Added Sugars." Accessed April 15, 2020. https://www.cdc.gov/nutrition/data-statistics/knowyour-limit-for-added-sugars.html

Duyff, R. L. 2012. American Dietetic Association Complete Food \& Nutrition Guide, 4th Edition. New York: Houghton Mifflin Harcourt Publishing Company. 55-87.

U.S. Food and Drug Administration (FDA). 2018. "Additional Information about High-Intensity Sweeteners Permitted for Use in Food in the United States." Accessed April 15, 2020. https://www.fda.gov/Food/IngredientsPackagingLabeling/FoodAdditivesIngredients/ucm397725.htm

U.S. Food and Drug Administration (FDA). 2019. "Generally Recognized as Safe (GRAS).” Accessed January 22, 2020. https://www.fda.gov/Food/IngredientsPackagingLabeling/GRAS/default.htm

Mahan, L. K., and S. Escott-Stump. 2008. Krause's Food \& Nutrition Therapy, 12th Edition. St. Louis, MO: Saunders Elsevier. 43.

Raben, A., A. C. Møller, T. H. Vasilaras, and A. Astrup. 2001. "A Randomized 10 Week Trial of Sucrose vs Artificial Sweeteners on Body Weight and Blood Pressure After 10 Weeks." Obesity Research 9:86s.

Reid, M., R. Hammersley, and M. Duffy. 2010. "Effects of Sucrose Drinks on Macronutrient Intake, Body Weight, and Mood State in Overweight Women over 4 Weeks." Appetite 55 (1): 130-136. https://doi.org/10.1016/j.appet.2010.05.001

Reid, M., R. Hammersley, M. Duffy, and C. Ballantyne. 2013. "Effects on Obese Women of the Sugar Sucrose Added to the Diet over 28 D: A Quasi-Randomised, SingleBlind, Controlled Trial." British Journal of Nutrition 111 (3): 563-570. https://doi.org/10.1017/S0007114513002687

Reid, M., R. Hammersley, A. J. Hill, and P. Skidmore. 2007. "Long-Term Dietary Compensation for Added Sugar: Effects of Supplementary Sucrose Drinks over a 4-Week Period." British Journal of Nutrition 97 (1): 193-203. https:// doi.org/10.1017/S0007114507252705
Ruiz-Ojeda, F. J., J. Plaza-Diaz, M. J. Saez-Lara, and A. Gil. 2019. "Effects of Sweeteners on the Gut Microbiota: A Review of Experimental Studies and Clinical Trials." Advances in Nutrition 10 (suppl_1): S31-S48. https://doi. org/10.1093/advances/nmy037

Toews, I., S. Lohner, D. Küllenberg de Gaudry, H. Sommer, and J. J. Meerpohl. 2019. "Association between Intake of Non-sugar Sweeteners and Health Outcomes: Systematic Review and Meta-analyses of Randomised and Nonrandomised Controlled Trials and Observational Studies." BMJ 364:k4718. https://doi.org/10.1136/bmj.k4718

United States Department of Agriculture (USDA). 2015. "Dietary Guidelines for Americans 2015-2020." Accessed April 15, 2020. http://health.gov/dietaryguidelines/2015/ guidelines/

United States Department of Agriculture (USDA). n.d. "FoodData Central." Accessed April 15, 2020. https://fdc. nal.usda.gov/index.html 